\title{
Sobrevivência de larvas de anisakídeos de peixe espada, Trichiurus lepturus (L.), submetidos aos processos de salmouragem e cocção
}

\section{Survival of anisakids larvae of cutlass fish, Trichiurus lepturus (L.), submitted to brine and cooking processes}

\author{
Sérgio Carmona de São Clemente ${ }^{1}$, Cristiane Miranda da Silva² ${ }^{2}$ Fabiana Pizzolato de Lucena ${ }^{2}$
}

\section{Resumo}

Um total de 40 espécimes de peixe espada, Trichiurus lepturus, foram analisados para se observar a sobrevivência de larvas de anisakídeos submetidos à salmoura e cocção. Os parasitos coletados pertenciam aos gêneros Contracaecum, Phocanema e Anisakis com prevalências de $95,5 \%, 3,8 \%$ e $0,7 \%$, respectivamente. O efeito da salmoura a $20^{\circ}$ Bé (Baumé) na sobrevivência das larvas demonstrou que, ao final de $72 \mathrm{~h}$ de exposição, todas encontravam-se mortas. Nos peixes submetidos à cocção a temperatura de $100^{\circ} \mathrm{C}, 5,9 \%$ das larvas permaneceram vivas ao final de $30 \mathrm{~min}$. e todas morreram ao final de $60 \mathrm{~min}$. Estes resultados sugerem que o melhor método para matar larvas de anisakídeos em peixe espada é a cocção.

Palavras-chave: anisakídeos; Trichiurus lepturus; cocção; salmoura

\section{Introdução}

A presença de larvas de anisakídeos em pescado marinho utilizado como alimento tem sido uma preocupação em todo o mundo, por elas constituirem um potencial problema de saúde pública, como também um problema estético. O primeiro caso de anisakiose humana foi descrito por Van Thiel et al. (1960). Após esse relato, vários registros têm sido feitos em diferentes partes do mundo. Sprent (1969) classificou a anisakiose humana como uma antropozoonose de ampla distribuição geográfica, ocorrendo principalmente em regiões próximas ao litoral, devido à facilidade de consumo dos produtos do mar. Em função dos hábitos alimentares foi evidenciado, pelas estatísticas, que a casuística se apresentou mais freqüente naqueles países onde o consumo de produtos da pesca ocorre sob determinadas condições: crus, mal cozidos, defumados a frio, inadequadamente salgados e re frigerados (Margolis, 1977; Yoshimura et al., 1979; Lagoin, 1980; Julini, 1993; Roepstorff et al., 1993).

Diferentes métodos vêm sendo empregados na tentativa de inviabilizar larvas de anisakídeos em peixes marinhos. Margolis (1977) registra que o cozimento a $70^{\circ} \mathrm{C}$ por $7 \mathrm{~min}$. e a $60^{\circ} \mathrm{C}$ por $10 \mathrm{~min}$. foram suficientes para matar larvas de Anisakis presentes em filés de peixes com $30 \mathrm{~mm}$ de espessura. Bier (1976) descreve que, em filés de pescado, a temperatura de $60^{\circ} \mathrm{C}$ foi suficiente para matar larvas de anisakídeos em 1 min. Grabda (1991) assinala que 10 dias foram o bastante para matar as larvas de Anisakis em arenques mantidos em salmoura a $20^{\circ}$ Bé. Karl e Leineman (1981) relatam que foram necessários 21 dias para matar todas as larvas de anisakídeos em arenques salgados a uma concentração de $20 \%$ de sal.

\section{Material e Métodos}

Entre setembro e novembro de 1995, 40 espécimes de $T$. lepturus foram adquiridas de pescadores profissionais do litoral de Niterói, Estado do Rio de Janeiro. Os peixes coletados foram acondicionados em caixas de isopor contendo gelo e transferidos para o Laboratório de Higiene e Tecnologia de Pescado da Faculdade de Veterinária da Universidade Federal Fluminense.

- Os peixes foram separados em dois lotes: o primeiro foi dividido em quatro grupos de cinco, mantidos em salmoura a $20^{\circ}$ Bé por $12,24,48$ e $72 \mathrm{~h}$. O segundo lote foi dividido em dois grupos de dez peixes que foram submetidos à temperatura de cocção a $100^{\circ} \mathrm{C}$, em forno de cozimento, durante 30 e 60 min., respectivamente. Ao final de cada experimento os peixes foram necropsiados e as larvas separadas em vivas e mortas. A sobrevivência das larvas posteriormente identificadas, foi observada através de movimento espontâneo e/ou estímulo mecânico.

1. Departamento de Tecnologia dos Alimentos, Faculdade de Veterinária, Universidade Federal Fluminense (UFF), Rua Vital Brazil Filho 64, 24230-340 Niterói, RJ, Brasil. Bolsista Pesquisador - CNPq

2 Bolsista Iniciação Científica PIBIC - CNPq/UFF 


\section{Resultados}

Foram examinados 40 espécimes de $T$. lepturus, todos positivos para anisakídeos. As larvas coletadas pertenciam aos gêneros Contracaecum, Phocanema e Anisakis, onde observou-se prevalência de 95,5\%,3,8\% e $0,7 \%$, respectivamente. A localização destas larvas estava restrita à serosa das vísceras e cavidade abdominal.

O efeito da salmoura na sobrevivência das larvas de anisakídeos demonstrou que $70 \%$ estavam vivas nas primeiras $12 \mathrm{~h}$ e que todas estavam mortas ao final de $72 \mathrm{~h}$ (Tabela 1).

Tabela 1 - Percentual de sobrevivência de larvas de anisakídeos de Trichiurus lepturus, mantidos em salmoura a $20^{\circ}$ Bé

\begin{tabular}{cccrc}
\hline Tempo de & Peixes & Anisakídeos & \multicolumn{2}{c}{ Anisakídeos } \\
salmouragem $(h)$ & examinados & coletados & vivos & $\%$ \\
\hline 12 & 5 & 1.520 & 1.067 & 70,0 \\
24 & 5 & 284 & 22 & 7,7 \\
48 & 5 & 370 & 7 & 1,9 \\
72 & 5 & 286 & 0 & 0 \\
\hline
\end{tabular}

Nos 20 peixes submetidos à temperatura de cocção, observou-se, ao final de $30 \mathrm{~min}$., que $5,9 \%$ das larvas coletadas permaneceram vivas e que $60 \mathrm{~min}$. foram suficientes para matar todas as larvas (Tabela 2).

Tabela 2 - Percentual de sobrevivência de larvas de anisakídeos de Trichiurus lepturus submetidos à temperatura de cocção $\left(100^{\circ} \mathrm{C}\right)$

\begin{tabular}{cccrc}
\hline Tempo de & Peixes & Anisakídeos & \multicolumn{2}{c}{ Anisakídeos } \\
cocção (min.) & examinados & coletados & vivos & $\%$ \\
\hline 30 & 10 & 327 & 19 & 5,2 \\
60 & 10 & 244 & 0 & 0 \\
\hline
\end{tabular}

Com relação à sobrevivência dos gêneros de anisakídeos submetidos à salmoura e à cocção, somente as larvas do gênero Contracaecum sobreviveram à cocção por até $30 \mathrm{~min}$. e à salmoura por até $48 \mathrm{~h}$.

\section{Discussão}

No Brasil, não se conhece, até o momento, pesquisas relacionadas com a utilização da salmoura e da cocção visando a comprovação da sobrevivência de anisakídeos em peixes teleósteos.

A sobrevivência de larvas de Anisakis encontradas em filés de peixes marinhos submetidos à cocção foi testada por Margolis (1977) e Bier (1976).

A diferença dos resultados apresentados pelos autores e os do presente trabalho, pode estar relacionada com o tipo de produto testado, uma vez que eles utilizaram somente filés de peixe e não peixes inteiros.

Com relação à utilização de salmoura, Karl e Leineman (1989) necessitaram de 21 dias para matar todas as larvas de anisakídeos presentes em arenques (Clupea harengus) e Grabda (1991) observou que 10 dias foram suficientes para obter o mesmo resultado na mesma espécie de peixe.

Comparando nossos resultados com os dos autores citados, 3 dias foram suficientes para inativar todas as larvas de anisakídeos encontradas nos peixes espada submetidos à salmoura.

Os resultados obtidos no presente trabalho permitem concluir que o melhor método a ser utilizado para matar larvas de anisakídeos em peixe espada é a cocção.

\section{Abstract}

\section{Survival of anisakids larvae of cutlass fish, Trichiurus lepturus (L.), submitted to brine and cooking processes}

Fourty specimens of cutlass fish Trichiurus lepturus were analyzed in order to observe the survival level of the anisakids larvae submitted to brine and cooking processes. The collected parasites were of the genera Contracaecum, Phocanemaand Anisakis with a prevalence of $95.5 \%, 3.8 \%$ and $0.7 \%$, respectively. All larvae died after being exposed to $72 \mathrm{hr}$ of brine at $20^{\circ}$ Bé. In cooked fishes at $100^{\circ} \mathrm{C}, 5.9 \%$ of larvae remained alive after $30 \mathrm{~min}$, and all of them died after $60 \mathrm{~min}$. These results suggest that cooking is the best method to kill anisakids larvae in cutlass fish.

Key words: anisakids; Trichiurus lepturus; cooking; brine

\section{Referências bibliográficas}

BIER, J. W. Experimental anisakiasis: Cultivation and temperature tolerance determinations. J. Milk Food Technol., v. 39, n. 2, p. 132-139, 1976.

GRABDA, J. Marine fish parasitology: an outline. PWN - Polish Scientific Publishers, Warszawa. 1991, 306 p.

JULINI, M. Anisakidosi. Nu. Prog. Vet. v. 47, n. 2, p. 54-56, 1993.

KARL, H., LEINEMANN, M. Überlebensfähigkeit von nematodenlarven bei der herstellung von gesalzenen heringserzeugnissen. Archiv für Lebensmittelhyg., v. 40, p. 107-106, 1989.

LAGOIN, Y. Donnés actuelles sur une nematodes larvaire de l'homme, l'anisakiase on "maladie ou ver du hareng". Bull. Acad. Vet. de France, v. 53, p. 139-146, 1980.

MARGOLIS, L. Public health aspects of "Codworm Infection". J. Fish Res. Bd Can., v. 34, n. 7, p. 887-898, 1977.

ROESTORFF, A., KARL, H., BLOEMSMA, B., HUSS, H.H. Catch handing and the possile migration of Anisakis larvae in Herring, Clupea harengus. J. Food Protection, v. 56, n. 9, p. 783-787, 1993.

SPRENT, J.F.A. Helminth "Zoonoses": An Analysis. Helminthol. Abst., v. 38, p. 333-351, 1969.

VAN THIEL, P.H., KUIPERS, C.F., ROSKAN, T. H. A nematode parasitic to herring causing acute abdominal syndrome in man. Tropic Geog. Med., v. 12, p. 97-113, 1960.

YOSHIMURA, H., KONDO,K., AKAD, N., OHVISHI, Y., WATANABE, K., SHINNO, B., AIKAWA, K. Two cases of eosinophilic granulomas formes in the large omentum and mesentery by the penetrated Anisakis larva through the gastrointestinal tract. Stom. Intest., v. 14, p. 519-522, 1979. 